Meta

Journal des traducteurs

Translators' Journal

\title{
L'interprétation, manifestation élémentaire de la traduction
}

\section{Marianne Lederer}

Volume 30, numéro 1, mars 1985

Interprétation de conférence

URI : https://id.erudit.org/iderudit/003440ar

DOI : https://doi.org/10.7202/003440ar

Aller au sommaire du numéro

Éditeur(s)

Les Presses de l'Université de Montréal

ISSN

0026-0452 (imprimé)

1492-1421 (numérique)

Découvrir la revue

Citer cet article

Lederer, M. (1985). L’interprétation, manifestation élémentaire de la traduction. Meta, 30(1), 25-29. https://doi.org/10.7202/003440ar d'utilisation que vous pouvez consulter en ligne.

https://apropos.erudit.org/fr/usagers/politique-dutilisation/ 


\title{
L'INTERPRÉTATION, MANIFESTATION ÉLÉMENTAIRE DE LA TRADUCTION
}

\author{
MARIANNE LEDERER
}

La linguistique structurale a établi une distinction entre langue et "parole" mise en cuvre de la langue, classant dans cette dernière toutes les manifestations de la communication humaine, orales ou écrites, et réservant à la première l'objet qu'est le français, l'allemand, l'anglais, etc., dont on peut décrire l'état ou le fonctionnement indépendamment de toute communication. La grammaire générative a développé la notion de langue au profit de celle de compétence linguistique, capacité qu'a tout autochtone de distinguer ce qui se dit de ce qui ne se dirait pas dans sa langue. La théorie de l'interprétation ne s'intéresse ni à la langue saussurienne ni à la compétence chomskyenne ; les langues existent, l'interprète les maîtrise; elle s'intéresse à la situation de communication où se manifeste uniquement la parole ou, si l'on préfère, la performance. La parole, la langue plus l'individu, est le résultat conjugué du fonctionnement d'une langue et de celui des mécanismes cérébraux d'un individu.

\section{ANTÉRIORITÉ DE L'ORAL}

L'anthropologie situe à 150000 ans avant notre ère l'époque où « le langage ne devait pas différer de beaucoup du langage tel qu'il est connu chez les hommes actuels "; il assurait probablement " la communication au cours des actes (... et) aussi la transmission différée des symboles de l'action, sous forme de récits $" 1$.

La parole liée à l'activité technique des ancêtres de l'homme contemporain est apparue longtemps avant l'écriture : "Vers 1500 avant notre ère, les premiers alphabets consonantiques apparaissent en Phénicie, vers 750 les alphabets à voyelles sont installés en Grèce $»^{2}$. C'est dire l'antériorité de l'oral sur l'écrit.

L'écriture en donnant une forme graphique à la parole a fixé les langues, en a permis l'étude. Celle-ci, s'appuyant sur l'écrit que sa permanence rendait seul accessible, a perdu de vue le fonctionnement de l'oral malgré son antériorité. La linguistique contemporaine a essentiellement pris pour objet d'intérêt les formes figées des langues, leurs structures, morphologie et syntaxe, et leur sémantisme, laissant largement de côté l'emploi individuel du langage, caractérisé principalement par sa forme orale, le discours.

\section{DIFFÉRENCE DE RÉMANENCE ENTRE L'ÉCRIT ET L'ORAL}

Comme les théories du langage, les théories de la traduction ont quasiment toutes en commun le fait d'être bâties sur l'écrit. La stabilité des signes figés sur le papier retient l'attention et l'examen de deux textes écrits, l'original et la traduction, porte tout naturellement à étudier les deux produits et donc à comparer les langues, sans s'intéresser à la façon dont il a été procédé pour réaliser la traduction.

C'est un fait que si l'on passe en revue les ouvrages sur la traduction, on s'aperçoit qu'ils tournent autour de problèmes linguistiques (intraduisibilité de certains termes, différences syntaxiques, ambiguïtés qui ne trouvent pas à se perpétuer sous un terme de 
l'autre langue...) et ce n'est qu'en toute dernière analyse, lorsqu'on aborde la typologie des textes, qu'est évoqué le problème de méthode : traduit-on un texte littéraire comme on traduit une information, un texte philosophique comme une publicité?

La parole orale de son côté qui a été pendant des millénaires le seul caractère distinctif de l'humanité, n'a que fort peu attiré les chercheurs, en dehors de ses aspects vocaux dont l'étude a donné naissance aux sciences de la phonétique et de la phonologie et à certaines recherches sur la prosodie. Il semble admis que l'oral, langage brut et approximatif comparé à l'écrit, ne peut se prêter à des pensées profondes ; malgré la place toujours plus grande que prend l'audio-visuel dans la vie contemporaine, on considère encore que seul ce qui est écrit est sérieux, probablement parce que ce qui est écrit reste, et que la transmission du savoir s'effectue grâce à lui.

Sans doute faut-il l'expérience de l'interprète de conférence pour savoir à quel point les idées qu'expriment lorsqu'ils se parlent les chercheurs comme les politiques, les industriels comme les artistes dépassent, et de loin, les banalités de la conversation trop souvent prise pour modèle du langage oral. C'est aussi l'interprète qui peut démontrer par ses prestations que la forme sonore de l'oral qui s'évanouit en quelques secondes, marque le cerveau d'une trace cognitive durable.

\section{LA TRACE DE L'ORAL}

Les sons disparaissent quelques secondes après leur émission ; les mots, les phrases s'envolent les uns après les autres. Les interlocuteurs en retirent néanmoins une compréhension qui leur permet de suivre sans peine le sens des paroles échangées, de porter la contradiction ou de renchérir sur les propos énoncés... Rares sont les mots dont ils conservent la forme en mémoire ; il arrive certes qu'un mot agisse en détonateur par l'indication qu'il fournit d'une appartenance politique ou d'une conviction idéologique et que sa forme reste en mémoire, mais dans l'ensemble la trace de l'oral est cognitive.

S'il est exact que nul ne peut répéter des phrases entendues d'affilée pendant quelques minutes, l'interprète de consécutive prouve que leurs contenus cognitifs peuvent être retenus en une seule audition dans leurs détails et leurs nuances. Nulle polysémie, nulle ambiguïté n'entrave sa compréhension. Les paroles dévêtues de leur enveloppe signifiante sont univoques ; pour l'interprète, l'évanescence des formes est un don du ciel ; leur fugitivité même lui évite toute interrogation sur ce qu'elles pourraient signifier par ailleurs. Le contenu cognitif motive seul sa restitution. Par ailleurs, ne participant pas au débat qui se déroule dans la salle, n'ayant pas à se soucier ni de porter la contradiction ni de renchérir, il concentre intégralement son attention sur l'argumentation des protagonistes et ne laisse échapper aucune idée, quelles qu'en soient les subtilités.

Si complète que soit cette interprétation, elle repose sur une phase intermédiaire entre le discours original et sa restitution, une phase marquée par la déverbalisation qui se produit chaque fois qu'est dépassée la durée de l'empan auditif. Cette déverbalisation est si naturelle qu'en consécutive il arrive à l'interprète de ne pas prendre conscience de la langue dans laquelle une intervention a été faite et d'en commencer la "traduction" en s'exprimant dans cette langue ! L'interprète de consécutive maître de sa technique relate plus qu'il ne traduit.

L'interprète de simultanée en revanche n'est pas entièrement épargné par les difficultés qui assaillent la traduction de l'écrit, car les mots de l'original résonnent encore au moment où il opère sa traduction; s'il se laisse aller à les traduire en tant que tels, s'il oublie que la déverbalisation est indispensable à une restitution intelligible, il s'engluera dans le transcodage. 


\section{LA TRACE GRAPHIQUE}

Des formes sonores de la parole orale, il ne subsiste qu'une trace cognitive. L'écrit, dans sa graphie, conserve en plus la trace des signifiants. La permanence du sensible, la représentation des formes de la parole permet son analyse en phrases, en propositions, en marques grammaticales, etc. Certes, la perception visuelle du lecteur intéressé par le contenu d'un texte fonctionne de la même manière que sa perception auditive d'un discours ; il appréhende simultanément un certain nombre de signes qui se coagulent en "petits" sens, chacun d'eux s'intégrant aux suivants pour constituer le sens général du texte. Mais la parole écrite étant toujours disponible, elle autorise un ralentissement du rythme d'appréhension naturel du langage. Elle permet de s'attarder sur des groupes de syllabes ou de mots; l'on peut ainsi appréhender les significations multiples de mots ou de phrases. Ce type de saisie par empan restreint mène à la découverte de différentes couches de significations dans les phrases et les mots. La rémanence de l'écrit permettant cette lecture multiple peut conduire à différents niveaux de traduction : transphonation pure (to initiate $=$ initier, malicious $=$ malicieux...), transcodage de la motivation (Arbeitskraft $=$ force de travail), du sens premier (to browse = brouter avant faire $d u$ lèche-vitrine), calque syntaxique (My name is Miller and I come from Australia donnant Mon nom est Miller et je viens d'Australie au lieu de je m'appelle Miller, je suis Australien). Celui qui traduit concentré sur la langue ne fait jouer que sa compétence linguistique et oublie de mettre en jeu tous les autres facteurs qui contribuent au processus normal de la lecture des textes ; il ne faut pas appeler le produit de ce travail traduction mais transcodage; il faut réserver le terme noble de traduction à la transposition qui s'appuie sur l'appréhension cognitive des textes et réalise non un transfert de langue mais un transfert d'idées. Aussi une grande méfiance s'impose-t-elle à l'égard de l'écrit. Le bon traducteur, n'ayant pas la chance de voir disparaître les formes signifiantes à peine émises, et sa tâche n'étant pas facilitée par l'impossibilité de retenir autre chose que le contenu cognitif, que les idées du discours, doit faire front consciemment pour dépasser la trace graphique.

\section{TRADUCTION, INTERPRÉTATION, UN MÊME PROCESSUS}

La démarche psychologique de base de la compréhension ne peut être différente pour l'écrit et pour l'oral mais elle est plus facile à discerner en interprétation qu'en traduction. L'interprète travaille sur une matière orale qui laisse une trace déverbalisée; le fait même que les formes sonores, loin d'être figées noir sur blanc, cessent très rapidement de résonner à ses oreilles réduit la langue à sa juste place : elle n'est que l'un des divers facteurs qui contribuent à la compréhension. Les autres facteurs sont essentiellement mentaux; nous les appelons bagage et contexte cognitifs. Le premier est constitué des connaissances générales et thématiques sur fond desquelles l'interprète accueille les énoncés qui lui parviennent. Le second se construit à mesure que se déroulent les dialogues; l'accumulation du savoir que l'interprète partage avec les interlocuteurs facilite de plus en plus sa perception de chaque nouvelle idée qui, allant à son tour rejoindre son savoir, améliore encore la structure d'accueil des paroles.

La comparaison de nombreuses interprétations avec leurs originaux retenus sur bandes magnétiques montre la part de ces différents facteurs dans la compréhension.

Ce qui est vrai de l'interprète l'est de chacun de nous ; nul ne reçoit jamais une information dans un cerveau vide, ou ne contenant rien qu'une compétence linguistique. Toute perception est toujours reçue par un ensemble de ce que J. Piaget appelle des schèmes, qui assimilent les perceptions et s'y accommodent. La perception des énoncés linguistiques est traitée par le cerveau de façon analogue à toutes les autres perceptions qui viennent du monde qui nous entoure. 
Dans l'écrit, la langue semble seule présente et peut mobiliser entièrement les perceptions ; elle tend à occulter le rôle du bagage et du contexte cognitifs dans la compréhension. Or, ceux-ci comptent tout autant que dans l'oral : le bagage cognitif c'est tout ce que le traducteur doit savoir du milieu historique et social d'une ouvre, et tout ce qu'il doit connaître des techniques, des procédés ou de la science qu'il aborde ; le contexte cognitif joue le même rôle que dans l'oral, aucune ligne d'un texte n'étant pleinement comprise indépendamment des lignes qui la précèdent.

Par définition, la compréhension, mécanisme intellectuel, ne saurait fonctionner différemment selon le support qui présente le sens à assimiler.

La lecture orientée vers l'appréhension des contenus ne fonctionne pas différemment de l'écoute intelligente ; le lecteur ne retient pas la chaîne écrite mais les sens qui s'en dégagent. À mesure qu'il lit, les significations des mots se fondent les unes dans les autres et avec les connaissances préalables. Traducteurs et interprètes sont en premier lieu des lecteurs et des auditeurs, les objets de leur compréhension sont les mêmes : idées, contenus notionnels et émotionnels des textes et des discours.

\section{DEUX PRODUITS DIFFÉRENTS}

Interprètes et traducteurs comprennent selon la même démarche intellectuelle ce qui leur parvient, aux uns oralement, aux autres par écrit, mais ils le restituent différemment, respectant en cela les lois qui s'appliquent à leurs moyens d'expression respectifs. La même exigence d'intelligibilité détermine la différence entre les résultats qu'ils produisent.

Le produit écrit est une œuvre durable, et le traducteur, pour la rendre lisible, se modèle sur les normes de l'écriture. L'écriture vise un public vaste et souvent mal défini. Elle se donne le temps de chercher la rédaction la plus convenable ; elle respecte les lois de la syntaxe, craint les répétitions et construit le texte en propositions et en phrases. Elle supplée à l'absence de prosodie des signes typographiques par la précision et la richesse du vocabulaire. Aussi, le traducteur choisit-il délibérément son expression et la perfectionne si nécessaire par des retours en arrière et des retouches. La forme sur laquelle il travaille reste présente à ses yeux; il en pèse les termes, analyse chacun des traits du texte et vérifie la correspondance de sa version au sens et au style de l'original.

Le texte terminé abandonne son auteur pour vivre une existence autonome; le produit du traducteur fait de même.

Le produit oral est très vite débarrassé de son éphémère vecteur sonore. La parole orale, contrairement à la parole écrite, ne fait pas de halte dans son trajet de l'émetteur au récepteur. Le son des paroles s'évanouit et elles-mêmes ne subsistent que sous forme de souvenirs cognitifs dans la mémoire. Le rythme naturel de la parole est soutenu et bien plus rapide que celui de l'écriture. L'intelligibilité de l'oral n'est pas due à la segmentation grammaticale mais à la continuité d'unités de sens aux limites indéfinissables, qui s'allongent et se rétractent selon les réactions qu'elles déclenchent. La parole orale est redondante ; émise sur bruit de fond, elle serait parfois perdue si elle n'était répétitive. Les traits prosodiques transmettent des sens que l'écriture doit analyser en mots et en style, et le ton compense souvent le manque de propriété des termes.

L'interprète suit le mouvement de la parole. Les enregistrements sonores d'interprétations montrent l'extraordinaire rapidité de l'opération interprétative, résultat du contact nu et immédiat entre la pensée que l'interprète assimile à mesure qu'il l'entend, et sa propre parole dont la formulation obéit à des réflexes d'énonciation acquis depuis longtemps. Ces enregistrements montrent aussi que l'interprète s'exprime avec une totale spontanéité et qu'il parvient à se faire comprendre d'un seul jet; des formulations qui, mises par écrit, sembleraient pauvres et parfois incorrectes, sont remarquablement intelligibles car conformes aux lois de l'oral. 
L'orateur que l'interprète traduit a toujours un public et si, dans l'émission de paroles ses idées se chassent l'une l'autre, il s'adapte constamment à ses auditeurs. L'interprète s'adapte aux siens, sa formulation tient compte des différences de savoirs entre origines culturelles diverses. Lorsque l'orateur se tait, ses paroles s'évanouissent à jamais, sauf pour le sens qu'elles ont transmis. Il en va de même de l'interprétation.

J'ai publié ailleurs (Lederer 1981) la transcription de 60 minutes de discours oraux et de leurs interprétations. Pour une étude approfondie de l'interprétation simultanée, il était indispensable de fixer la forme des interprétations. Je me dispenserai de recommencer ici car la transcription de l'oral en écrit fausse l'observation du produit de l'interprétation, qui est purement cognitif, sans l'étape intermédiaire que connaît la parole écrite.

Par contre, le sort d'une traduction étant, comme celui du texte original, de perdurer, je reproduis ci-dessous un bref extrait de la traduction du livre de G. Steiner, $A f$ ter $\mathrm{Babel}^{3}$. On y verra que la démarche du bon traducteur est bien celle que nous avons indiquée, alors que son produit revêt une forme infiniment plus sophistiquée que dans l'oral.

\section{Texte original}

Chapter Four - The Claims of theory

"The literature on the theory, practice, and history of translation is large. It can be divided into four periods, though the lines of division are in no sense absolute. »

\section{Traduction}

$$
\begin{aligned}
& \text { Chapitre Quatre - Les ambitions de la } \\
& \text { théorie } \\
& \text { "Il ne manque pas d'ouvrages sur la } \\
& \text { théorie, la pratique et l'histoire } \\
& \text { de la traduction. On peut les } \\
& \text { regrouper en } 4 \text { périodes dont les lignes } \\
& \text { de démarcation n'ont cependant rien } \\
& \text { d'absolu." }
\end{aligned}
$$

Le titre du chapitre, The Claims of Theory, est traduit par Les ambitions de la théorie autant en fonction de la pensée de Steiner que de l'énoncé linguistique. Quant à la traduction des deux premières phrases de ce chapitre, on notera la remarquable élégance d'expression avec laquelle la version française interprète les énoncés originaux pour en dégager le sens.

Toute traduction est interprétation; la perfection de la forme écrite le démontre. Elle n'en révèle pas pour autant le processus qu'il est le privilège de la traduction orale, aux formes évanescentes, de faire apparaître.

Notes

1. A. Leroi-Gourhan (1964) : le Geste et la parole, I. Techniques et langage, Paris, Albin Michel, p 165.

2. Op. cit., p. 291.

3. G. Steiner (1975) : After Babel, New York, Oxford University Press.

RÉFÉRENCES

LEDERER, M. (1981) : la Traduction simultanée, Paris, Minard.

SELESKOVITCH, D. et M. LEDERER (1984) : Interpréter pour traduire, Paris, Didier Érudition. 